\title{
Safeguard global supply chains during a pandemic
}

\author{
A study of the grain trade during 2020 indicates that policies to protect supply chains must be enacted to avoid \\ supply chain shocks such as COVID-19 and locust swarms exacerbating food insecurity in global regions that rely \\ on food imports.
}

\section{Louise Manning}

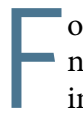
ood insecurity is complex - there is no silver bullet of policy or market intervention that can lead to a situation where all people at all times will have continuous access to healthy, affordable diets. And though global food systems are interdependent and also complex, food insecurity in many regions has been precipitated by pestilence, environmental disaster and conflict. Pestilence is a fatal epidemic or pandemic disease affecting humans, crops or livestock that impacts food supply and production; insect and rodent plagues remain a major threat to human food security ${ }^{1-5}$. Recently, swarms of locusts larger than any recorded in recent decades detrimentally affected more than 330,000 hectares of land from Ethiopia to India 6 , whilst the COVID-19 pandemic - and the controls implemented to curb infection rates - affected food production and supply.

In times of crisis, the demand for staple foods increases in ways that can destabilize local and global supply chains and cause social unrest $^{3,7}$. In this issue of Nature Food, Falkendal et al. ${ }^{8}$ quantify wheat, rice and maize supply chain disruption from 2020 locust swarms and COVID-19-related effects on food prices, stock levels, international trade and export restrictions. The study considers two dimensions of food security, first outlined nearly a quarter of a century ago at the World Food Summit in 1996, namely: physical availability of food (production output, stock levels and trade dynamics) and economic and physical access to food (the ability to buy food, for example, ratio of prices to income, and accessible marketing channels). The authors frame their argument in terms of stability and the socio-economic shocks (political instability, unemployment and drastic loss of income) that the COVID-19 pandemic brings with it that will lead to greater food insecurity in the short and medium term.

In their model, Falkendal and colleagues find that export restrictions and precautionary purchasing in response to COVID-19 could destabilize global grain trade, leading to many low- and middle-income countries that rely on grain imports potentially experiencing further food insecurity that exacerbates the effects felt from shocks such as COVID-19 and locust swarms. Thus, protectionist measures initiated by governments, institutions or market actors to secure national food security will affect those who are food vulnerable, and consumer support policy measures should be introduced to mitigate the risk of food insecurity. The authors call for incremental rather than blunt, binary 'borders open or borders closed' food security policies, and a need for mutually agreed solutions to address food insecurity - rather than unilateral national decision-making based primarily on self-interest. Whether altruist or self-serving food security policies are implemented by governments and market actors will be demonstrated in practice over the coming months.

The impact of economic stabilization policies following the 2007 economic crash highlights how individuals and households can transition instantly from a higher standard of living into a situation where they must survive with less, raising the question as to what is the minimum standard for an acceptable life ${ }^{9}$. In the UK, the last time minimum standards with regard to food for an acceptable life were determined was the food rationing legislation on 15 September $1941^{10}$ - the Hansard report makes challenging reading when comparing the proposed austere diet to our typical food consumption in the UK.
The UN Sustainable Development Goals also determine the dynamics of an acceptable life, and multi-level consensus building and action is essential to safeguard food supply - especially if, as a global community, we seek to deliver the two targets of "no poverty and zero hunger". Despite having policy and technological tools to reduce the impact of many human, zoonotic and plant diseases, collective strategic risk at local, regional and global levels cannot be ignored. Falkendal and colleagues have shown that a proactive strategy and a co-ordinated collective response with shared goals and co-operative actions is necessary as the combination of the COVID-19 pandemic and natural events such as locust swarms arise in order to ensure that the grain trade remains stable, equitable and accessible to all.

\section{Louise Manning (D) $₫$}

School of Agriculture, Food and Environment, Royal Agricultural University, Gloucestershire, UK.

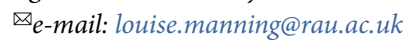

Published online: 13 January 2021 https://doi.org/10.1038/s43016-020-00213-5

References

1. Stenseth, N. C. et al. Front. Ecol. Environ. 1, 367-375 (2003).

2. Caughley, J., Monamy, V. \& Heiden, K. Impact of the 1993 Mouse Plague (GRDC, 1994).

3. Barrett, C. B. Nat. Food 1, 319-320 (2020).

4. Locust Hub (FAO, 2020); https://locust-hub-hqfao.hub.arcgis. $\mathrm{com} /$

5. Australian Mouse Alert (MouseAlert, 2020); https://www.feralscan. org.au/mousealert/

6. Desert Locust Bulletin (FAO, 3 July 2020); http://www.fao.org/ag/ locusts/common/ecg/2556/en/DL501e.pdf

7. Paveliuc-Olariu, C. Adv. Agric. Bot. 5, 174-178 (2013).

8. Falkendal, T. et al. Nat. Food https://doi.org/10.1038/s43016-02000211-7 (2021).

9. Knight, D. M. \& Stewart, C. Hist. Anthropol. 27, 1-18 (2016).

10. Rationed Foodstuffs (Hansard, 30 September 1941); https://api. parliament.uk/historic-hansard/written-answers/1941/sep/30/ rationed-foodstuffs

Competing interests

The author declares no competing interests. 